\title{
Front Matter: Volume 7640
}

, "Front Matter: Volume 7640," Proc. SPIE 7640, Optical Microlithography XXIII, 764001 (16 April 2010); doi: 10.1117/12.862757

SPIE. Event: SPIE Advanced Lithography, 2010, San Jose, California, United States 


\section{PROCEEDINGS OF SPIE}

\section{Optical Microlithography XXIII}

Mircea V. Dusa

Will Conley

Editors

23-25 February 2010

San Jose, California, United States

Sponsored by

SPIE

Cooperating Organization

SEMATECH Inc. (United States)

Published by

SPIE 
The papers included in this volume were part of the technical conference cited on the cover and title page. Papers were selected and subject to review by the editors and conference program committee. Some conference presentations may not be available for publication. The papers published in these proceedings reflect the work and thoughts of the authors and are published herein as submitted. The publisher is not responsible for the validity of the information or for any outcomes resulting from reliance thereon.

Please use the following format to cite material from this book:

Author(s), "Title of Paper," in Optical Microlithography XXIII, edited by Mircea V. Dusa, Will Conley, Proceedings of SPIE Vol. 7640 (SPIE, Bellingham, WA, 2010) Article CID Number.

ISSN 0277-786X

ISBN 9780819480545

Published by

SPIE

P.O. Box 10, Bellingham, Washington 98227-0010 USA

Telephone +1 3606763290 (Pacific Time) · Fax +1 3606471445

SPIE.org

Copyright (c) 2010, Society of Photo-Optical Instrumentation Engineers

Copying of material in this book for internal or personal use, or for the internal or personal use of specific clients, beyond the fair use provisions granted by the U.S. Copyright Law is authorized by SPIE subject to payment of copying fees. The Transactional Reporting Service base fee for this volume is $\$ 18.00$ per article (or portion thereof), which should be paid directly to the Copyright Clearance Center (CCC), 222 Rosewood Drive, Danvers, MA 01923. Payment may also be made electronically through CCC Online at copyright.com. Other copying for republication, resale, advertising or promotion, or any form of systematic or multiple reproduction of any material in this book is prohibited except with permission in writing from the publisher. The CCC fee code is 0277-786X/10/\$18.00.

Printed in the United States of America.

Publication of record for individual papers is online in the SPIE Digital Library.

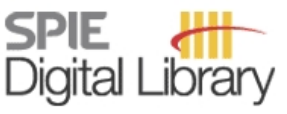

SPIEDigitalLibrary.org

Paper Numbering: Proceedings of SPIE follow an e-First publication model, with papers published first online and then in print and on CD-ROM. Papers are published as they are submitted and meet publication criteria. A unique, consistent, permanent citation identifier (CID) number is assigned to each article at the time of the first publication. Utilization of CIDs allows articles to be fully citable as soon they are published online, and connects the same identifier to all online, print, and electronic versions of the publication. SPIE uses a six-digit CID article numbering system in which:

- The first four digits correspond to the SPIE volume number.

- The last two digits indicate publication order within the volume using a Base 36 numbering system employing both numerals and letters. These two-number sets start with 00, 01, 02, 03, 04 , $05,06,07,08,09,0 A, 0 B \ldots$. OZ, followed by 10-1Z, 20-2Z, etc.

The CID number appears on each page of the manuscript. The complete citation is used on the first page, and an abbreviated version on subsequent pages. Numbers in the index correspond to the last two digits of the six-digit CID number. 


\section{Contents}

\section{Part One}

xvii Conference Committee

\section{SESSION 1 INVITED SESSION}

764003 Shaping the future of nanoelectronics beyond the Si roadmap with new materials and devices (Invited Paper) [7640-02]

M. Heyns, F. Bellenger, IMEC (Belgium) and Katholieke Univ. Leuven (Belgium);

G. Brammertz, M. Caymax, M. Cantoro, S. De Gendt, B. De Jaeger, A. Delabie, IMEC (Belgium); G. Eneman, IMEC (Belgium), Katholieke Univ. Leuven (Belgium), and FWO-Vlaanderen (Belgium); G. Groeseneken, G. Hellings, M. Houssa, IMEC (Belgium) and Katholieke Univ. Leuven (Belgium); F. Iacopi, D. Leonelli, D. Lin, W. Magnus, IMEC (Belgium); K. Martens, IMEC (Belgium), Katholieke Univ. Leuven (Belgium), and FWO-Vlaanderen (Belgium); C. Merckling, M. Meuris, J. Mitard, J. Penaud, G. Pourtois, M. Scarrozza, E. R. Simoen, B. Soree, S. Van Elshocht, IMEC (Belgium); W. Vandenberghe, IMEC (Belgium) and Katholieke Univ. Leuven (Belgium); A. Vandooren, P. Vereecke, A. Verhulst, W.-E. Wang, IMEC (Belgium)

\section{SESSION 2 FREEFORM AND SMO}

764005 Generation of arbitrary freeform source shapes using advanced illumination systems in high-NA immersion scanners [7640-04]

J. Zimmermann, P. Gräupner, J. T. Neumann, D. Hellweg, D. Jürgens, M. Patra, C. Hennerkes, M. Maul, Carl Zeiss SMT AG (Germany); B. Geh, Carl Zeiss SMT AG (Germany) and ASML US, Inc. (United States); A. Engelen, O. Noordman, M. Mulder, ASML (Netherlands); S. Park, J. De Vocht, Brion Technologies, Inc. (United States)

764006 Demonstrating the benefits of source-mask optimization and enabling technologies through experiment and simulations [7640-05]

D. Melville, A. E. Rosenbluth, IBM Thomas J. Watson Research Ctr. (United States); K. Tian, K. Lai, IBM Semiconductor Research and Development Ctr. (United States); S. Bagheri, IBM Thomas J. Watson Research Ctr. (United States); J. Tirapu-Azpiroz, J. Meiring, IBM Semiconductor Research and Development Ctr. (United States); S. Halle, G. Mclntyre, IBM Research at Albany Nanotech (United States); T. Faure, IBM Mask House (United States); D. Corliss, A. Krasnoperova, L. Zhuang, IBM Semiconductor Research and Development Ctr. (United States); P. Strenski, A. Waechter, L. Ladanyi, F. Barahona, D. Scarpazza, J. Lee, IBM Thomas J. Watson Research Ctr. (United States); T. Inove, M. Sakamoto, H. Muta, IBM Research Tokyo (Japan); A. Wagner, IBM Thomas J. Watson Research Ctr. (United States); G. Burr, Y. Kim, IBM Almaden Research Ctr. (United States); E. Gallagher, M. Hibbs, IBM Mask House (United States); A. Tritchkov, Y. Granik, M. Fakhry, K. Adam, G. Berger, M. Lam, A. Dave, N. Cobb, Mentor Graphics Corp. (United States) 
764007 Tolerancing analysis of customized illumination for practical applications of source and mask optimization [7640-06]

T. Matsuyama, N. Kita, T. Nakashima, O. Tanitsu, S. Owa, Nikon Corp. (Japan)

764008 Freeform illumination sources: an experimental study of source-mask optimization for 22-nm SRAM cells [7640-07]

J. Bekaert, B. Laenens, S. Verhaegen, L. Van Look, D. Trivkovic, F. Lazzarino,

G. Vandenberghe, IMEC vzW (Belgium); P. van Adrichem, ASML-BRION (United States) and

ASML (Netherlands); R. Socha, S. Baron, M. C. Tsai, K. Ning, S. Hsu, H. Y. Liu, ASML-BRION

(United States); M. Mulder, A. Bouma, E. van der Heijden, O. Mouraille, K. Schreel, J. Finders, M. Dusa, ASML (Netherlands); J. Zimmermann, P. Gräupner, J. T. Neumann, C. Hennerkes,

Carl Zeiss SMT AG (Germany)

\section{SESSION 3 DOUBLE PATTERNING I}

764009 Evaluation of double-patterning techniques for advanced logic nodes [7640-08]

C. Koay, S. Holmes, K. Petrillo, M. Colburn, S. Burns, IBM Corp. (United States); S. Dunn, Tokyo Electron Technology Ctr., America, LLC (United States); J. Cantone, Tokyo Electron America, Inc. (United States); D. Hetzer, S. Kawakami, Tokyo Electron Technology Ctr., America, LLC (United States); Y. van Dommelen, A. Jiang, M. Many, R. Routh, ASML (United States); L. Huli, B. Martinick, M. Rodgers, Univ. at Albany, SUNY (United States); H. Tomizawa, Toshiba (United States); S. Kini, KLA-Tencor (United States)

7640 OA Actual performance data analysis of overlay, focus, and dose control of an immersion scanner for double patterning [7640-09]

S. Wakamoto, Y. Kanaya, J. Kosugi, N. Kasai, H. Nishinaga, K. Shiraishi, Y. Shirata, Y. Ishii, Nikon Corp. (Japan)

7640 OB Modeling of double patterning interactions in litho-cure-litho-etch (LCLE) processes [7640-10]

A. Erdmann, F. Shao, Fraunhofer Institute for Integrated Systems and Device Technology (Germany); J. Fuhrmann, A. Fiebach, Weierstrass Institute for Applied Analysis and Stochastics (Germany); G. P. Patsis, Institute of Microelectronics (Greece); P. Trefonas, Dow Electronic Materials (United States)

$76400 \mathrm{C}$ Litho and patterning challenges for memory and logic applications at the 22-nm node [7640-11]

J. Finders, ASML (Netherlands); M. Dusa, ASML US Inc. (Netherlands); P. Nikolsky, Y. van Dommelen, ASML (Netherlands); R. Watso, ASML Albany (United States); T. Vandeweyer, J. Beckaert, B. Laenens, L. Van Look, IMEC vzW (Belgium)

7640 OD Comparative study of resolution limits for double patterning and EUV processes for the 32-nm contact hole case [7640-12]

I. Kamohara, K. Tajima, Nihon Synopsys GK (Japan); T. Schmoeller, Synopsys GmbH (Germany) 
7640 OE Advances in dual-tone development for pitch frequency doubling [7640-13]

C. Fonseca, M. Somervell, S. Scheer, Tokyo Electron America, Inc. (United States);

Y. Kuwahara, K. Nafus, Tokyo Electron Kyushu Ltd. (Japan); R. Gronheid, IMEC (Belgium);

S. Tarutani, Y. Enomoto, FUJIFILM Corp. (Japan)

7640 OF Spacer defined double patterning for sub-72-nm pitch logic technology [7640-14]

R. Kim, GLOBALFOUNDRIES Inc. (United States); E. Mclellan, Y. Yin, J. Arnold,

S. Kanakasabapathy, S. Mehta, IBM Research (United States); Y. Ma, GLOBALFOUNDRIES Inc. (United States); M. Burkhardt, IBM Research (United States); J. Cain, GLOBALFOUNDRIES Inc. (United States); G. Mclntyre, M. E. Colburn, IBM Research (United States); H. J. Levinson, GLOBALFOUNDRIES Inc. (United States)

7640 OG The impact of optical non-idealities in litho-litho-etch processing [7640-15] S. A. Robertson, KLA-Tencor Corp. (United States); M. T. Reilly, DOW Electronic Materials (United States); T. Graves, M. D. Smith, J. J. Biafore, KLA-Tencor Corp. (United States)

$7640 \mathrm{OH}$ Double patterning lithography study with high overlay accuracy [7640-16] T. Kikuchi, Y. Shirata, M. Yasuda, Y. Iriuchijima, K. Takemasa, R. Tanaka, A. Hazelton, Y. Ishii, Nikon Corp. (Japan)

7640 Ol Litho-process-litho for 2D 32-nm hp Logic and DRAM double patterning [7640-17] P. Wong, V. Wiaux, S. Verhaegen, N. Vandenbroeck, IMEC (Belgium)

7640 0J Modeling of exploration of reversible contrast enhancement layers for double exposure lithography [7640-18]

F. Shao, Fraunhofer Institute for Integrated Systems and Device Technology (Germany); G. D. Cooper, Z. Chen, Pixelligent Technologies LLC (United States); A. Erdmann, Fraunhofer Institute for Integrated Systems and Device Technology (Germany)

\section{SESSION 5 COMPUTATIONAL LITHOGRAPHY}

7640 OK Improving aberration control with application specific optimization using computational lithography [7640-19]

J. Zhou, Micron Technology, Inc. (United States); Y. Zhang, Brion Technologies (United States); P. Engblom, ASML (United States); M. Hyatt, Micron Technology, Inc. (United States); E. Wu, M. Snajdr, Brion Technologies (United States); A. deVilliers, Y. He, C. Hickman, Micron Technology, Inc. (United States); P. Liu, Brion Technologies (United States); D. de Lang, ASML Netherlands B.V. (Netherlands); B. Geh, Carl Zeiss SMT (United States); E. Byers, S. Light, Micron Technology, Inc. (United States)

$7640 \mathrm{OL} \quad$ Evaluation of lithographic benefits of using ILT techniques for 22 nm-node [7640-20] Y. Zou, Y. Deng, J. Kye, L. Capodieci, C. Tabery, GLOBALFOUNDRIES Inc. (United States); T. Dam, A. Aadamov, K.-H. Baik, L. Pang, B. Gleason, Luminescent Technologies, Inc. (United States)

7640 OM A computational method for optimal application specific lens control in microlithography [7640-21]

P. Liu, M. Snajdr, Z. Zhang, Y. Cao, J. Ye, Y. Zhang, Brion Technologies, Inc. (United States) 
7640 ON Aerial image calculation by eigenvalues and eigenfunctions of a matrix that includes source, pupil, and mask [7640-22]

K. Yamazoe, A. R. Neureuther, Univ. of California, Berkeley (United States)

764000 Optimization from design rules, source and mask, to full chip with a single computational lithography framework: level-set-methods-based inverse lithography technology (ILT) [7640-23]

L. Pang, D. Peng, P. Hu, D. Chen, T. Cecil, L. He, G. Xiao, V. Tolani, T. Dam, K.-H. Baik,

B. Gleason, Luminescent Technologies, Inc. (United States)

7640 OP Quadratic blur kernels for latent image formation modeling [7640-24]

A. Burov, Shanghai Microelectronics Equipment Corp. (China)

\section{SESSION 6 POLARIZATION}

$7640 \mathrm{OQ}$ In-situ Mueller matrix polarimetry of projection lenses for 193-nm lithography [7640-25]

H. Nomura, I. Higashikawa, Toshiba Corp. (Japan)

7640 OR Experimental result of polarization characteristics separation method [7640-26]

T. Fujii, K. Suzuki, J. Kogo, K. Toyama, K. Sasada, Nikon Corp. (Japan); M. Sawada, Nikon Systems Inc. (Japan)

\section{SESSION 7 BEYOND 22 NM}

7640 OS Implementing and validating double patterning in 22-nm to $16-\mathrm{nm}$ product design and patterning flows [7640-27]

M.-S. Noh, B.-S. Seo, S.-J. Lee, SAMSUNG Electronics Co., Ltd. (Korea, Republic of);

A. Miloslavsky, Synopsys (United States); C. Cork, Synopsys SARL (France); L. Barnes, Synopsys

Technology Park (United States); K. Lucas, Synopsys Inc. (United States)

7640 OT Comparative study of line width roughness (LWR) in next-generation lithography (NGL) processes [7640-28]

K. Patel, SanDisk (United States) and Univ. of California, Berkeley (United States); T. Wallow, H. J. Levinson, GLOBALFOUNDRIES Inc. (United States); C. J. Spanos, Univ. of California, Berkeley (United States)

\section{SESSION 8}

\section{TOOLS AND PROCESS RESOLUTION EXTENSIONS I}

7640 OU Toward perfect on-wafer pattern placement: stitched overlay exposure tool characterization [7640-29]

C. P. Ausschnitt, T. A. Brunner, N. M. Felix, IBM SRDC (United States); B. Minghetti, STMicroelectronics (France)

7640 OV Impact of scanner signatures on optical proximity correction [7640-30]

J. K. Tyminski, Nikon Precision Inc. (United States); T. Matsuyama, Nikon Corp. (Japan); Y.-L. LU, J.-C. Lai, K.-T. Chen, Y.-C. Mai, Powerchip Semiconductor Corp. (Taiwan); I. SU, Nikon (Japan); G. Bailey, Synopsys Taiwan Ltd. (Taiwan) 
7640 OW Overlay characterization and matching of immersion photoclusters [7640-31]

B. Minghetti, STMicroelectronics (United States); T. Brunner, C. Robinson, C. Ausschnitt,

D. Corliss, N. Felix, IBM SRDC (United States)

7640 OX Topcoat-less resist approach for high volume production and yield enhancement of immersion lithography [7640-32]

K. Nakano, R. Seki, T. Kawakubo, Y. Maruta, T. Sekito, K. Shiraishi, T. Sei, T. Fujiwara, T. Hayashi,

Y. Iriuchijima, S. Owa, Nikon Corp. (Japan)

7640 OY Analysis of the impact of pupil shape variation by pupil fit modeling [7640-33] J. Jeon, C. Park, H. Yang, C. Kim, J. Choi, S. Oh, D. Yim, S. Park, Hynix Semiconductor Inc. (Korea, Republic of); K. Park, Y. Min, ASML Korea (Korea, Republic of); A. Engelen, B. Smeets, ASML Netherlands B.V. (Netherlands); J. Zimmermann, Carl Zeiss SMT AG (Germany)

\section{SESSION 9 TOOLS AND PROCESS RESOLUTION EXTENSIONS II}

$7640 \mathrm{OZ} \quad$ Predicting and reducing substrate induced focus error [7640-34]

B. Liegl, B. Sapp, K. Low, S. Greco, T. Brunner, N. Felix, I. Stobert, K. Nafisi, IBM Corp. (United States); C. Sarma, Infineon Technologies (United States)

764010 Lithographic scanner stability improvements through advanced metrology and control [7640-35]

P. Vanoppen, T. Theeuwes, H. Megens, H. Cramer, T. Fliervoet, M. Ebert, D. Satriasaputra, ASML (Netherlands)

764011 Printing the metal and contact layers for the 32- and 22-nm node: comparing positive and negative tone development process [7640-69]

L. Van Look, J. Bekaert, V. Truffert, V. Wiaux, F. Lazzarino, M. Maenhoudt, G. Vandenberghe, IMEC (Belgium); M. Reybrouck, FUJIFILM Electronic Materials (Japan); S. Tarutani, FUJIFILM Corp. (Japan)

764013 The impacts of scanner modeling parameters for OPC model of sub-40-nm memory device [7640-116]

C. Kim, J. Jang, J. Jeon, C. Park, H. Yang, D. Yim, S. Park, Hynix Semiconductor Inc. (Korea, Republic of)

764014 Simulation-based pattern matching using scanner metrology and design data to reduce reliance on CD metrology [7640-38]

Y. He, E. Byers, S. Light, D. Hines, A. Devilliers, M. Hyatt, J. Zhou, V. Nair, Micron Technology, Inc. (United States); Z. Yu, Y. Cao, X. Xie, W. Shao, R. Aldana, R. Goossens, C.-Q. Ma, J. Lu, H. Liu, C. Aquino, Brion Technologies, Inc. (United States); P. Engblom, T. Nooitgedagt, E. Janda, ASML (United States)

764015 The GridMapper challenge: how to integrate into manufacturing for reduced overlay error [7640-39]

A. Gabor, B. Liegl, M. Pike, E. Hwang, T. Wiltshire, IBM Systems and Technology Group (United States) 
764016 Simultaneous optimization of dose and focus controls in advanced Arf immersion scanners [7640-40]

T. Toki, Nikon Corp. (Japan); P. Izikson, KLA-Tencor Corp. Israel (Israel); J. Kosugi, N. Sakasai,

K. Saotome, K. Suzuki, Nikon Corp. (Japan); D. Kandel, KLA-Tencor Corp. Israel (Israel);

J. C. Robinson, KLA-Tencor Corp. (United States); Y. Koyanagi, KLA-Tencor Japan (Japan)

\section{SESSION 10 MASK, LAYOUT, AND OPC}

764017 Novel fine-tuned model-based SRAF generation method using coherence map [7640-41]

K. Kodera, S. Tanaka, M. Yamaji, Toshiba Corp. (Japan); C. Kodama, Toshiba Memory Systems Co., Ltd. (Japan); T. Kotani, S. Nojima, K. Hashimoto, S. Mimotogi, S. Inove, Toshiba Corp. (Japan)

764018 Ultimately accurate SRAF replacement for practical phases using an adaptive search algorithm based on the optimal gradient method [7640-42]

S. Maeda, Toshiba Corp. (Japan); H. Nosato, National Institute of Advanced Industrial Science and Technology (Japan); T. Matsunawa, M. Miyairi, S. Nojima, S. Tanaka, Toshiba Corp. (Japan); H. Sakanashi, M. Murakawa, National Institute of Advanced Industrial Science and Technology (Japan); T. Saito, Toshiba Microelectronics Corp. (Japan); T. Higuchi, National Institute of Advanced Industrial Science and Technology (Japan); S. Inove, Toshiba Corp. (Japan)

764019 22-nm logic lithography in the presence of local interconnect [7640-43] M. C. Smayling, Tela Innovations, Inc. (United States); R. J. Socha, M. V. Dusa, ASML (United States)

7640 IA Lithography and layout co-optimization beyond conventional OPC concept [7640-44] K. Tsujita, K. Mikami, H. Ishii, T. Arai, K. Takahashi, Canon Inc. (Japan)

7640 1B Mask enhancer technology for sub-100-nm pitch random logic layout contact hole fabrication [7640-45]

T. Yuito, H. Sakaue, T. Matsuda, T. Shimizu, S. Irie, F. Iwamoto, A. Misaka, T. Koizumi, M. Sasago, Semiconductor Co., Panasonic Corp. (Japan)

7640 1C Suppressing ringing effects from very strong off-axis illumination with novel OPC approaches for low k1 lithography [7640-46]

C. Cork, Synopsys SARL (France); F. Amoroso, A. Poonawala, S. Jang, K. Lucas, Synopsys Inc. (United States)

\section{SESSION 11 MODELING}

7640 1D Three-dimensional physical photoresist model calibration and profile-based pattern verification [7640-47]

M. Talbi, A. Y. Abdo, T. C. Bailey, IBM Corp. (United States); W. Conley, Freescale Semiconductor, Inc. (United States); D. N. Dunn, IBM Corp. (United States); M. Fujimoto, NEC Electronics (United States); J. Nickel, IBM Corp. (United States); N. Y. Chung, SAMSUNG Electronics Co., Ltd. (Korea, Republic of); S. Marokkey, Infineon Technologies (United States); S. H. Lee, SAMSUNG Electronics Co., Ltd. (Korea, Republic of); C. Sarma, Infineon Technologies (United States); D. Shao, R. Viswanathan, IBM Corp. (United States) 
$7640 \mathrm{IE}$ The feasibility of using image parameters for test pattern selection during OPC model calibration [7640-48]

A. Abdo, R. Viswanathan, IBM Systems and Technology Group (United States)

$7640 \mathrm{IF}$ Optical proximity correction enhancement by using model based fragmentation approaches [7640-49]

Y.-S.Woo, W.-H. Choi, B.-S. Seo, Y. Kim, SAMSUNG Electronics Co., Ltd. (Korea, Republic of); V. Liubich, S. Abdelwahed, J. Kim, J. Word, Mentor Graphics Corp. (United States); J.-W. Lee, Mentor Graphics Korea (Korea, Republic of)

7640 1G Automation of sample plan creation for process model calibration [7640-50]

J. Oberschmidt, IBM Microelectronics (India); A. Abdo, IBM Microelectronics (United States);

T. Desouky, M. Al-Imam, Mentor Graphics Corp. (Egypt); A. Krasnoperova, R. Viswanathan,

IBM Microelectronics (United States)

\section{SESSION 12 SOURCE-MASK OPTIMIZATION}

$7640 \mathrm{1H} \quad$ SMO for 28-nm logic device and beyond: impact of source and mask complexity on lithography performance [7640-51]

S. Nagahara, K. Yoshimochi, H. Yamazaki, K. Takeda, T. Uchiyama, NEC Electronics Corp. (Japan); S. Hsu, Z. Li, H. Liu, K. Gronlund, T. Kurosawa, J. Ye, L. Chen, H. Chen, Z. Li, X. Liu, W. Liu, ASML Brion Technologies, Inc. (United States)

$764011 \quad$ Illumination optics for source-mask optimization [7640-52]

Y. Mizuno, T. Matsuyama, S. Owa, O. Tanitsu, N. Kita, M. Okumura, Nikon Corp. (Japan)

$7640 \mathrm{lJ}$ Considerations in source-mask optimization for logic applications [7640-53]

Y. Deng, Y. Zou, K. Yoshimoto, Y. Ma, C. E. Tabery, J. Kye, L. Capodieci, H. J. Levinson, GLOBALFOUNDRIES Inc. (United States)

$76401 \mathrm{~K}$ Challenges for low-k1 lithography in logic devices by source mask co-optimization [7640-54]

K. Yoshimochi, S. Nagahara, K. Takeda, T. Uchiyama, NEC Electronics Corp. (Japan)

7640 1L A GPU-based full-chip source-mask optimization solution [7640-55]

I. Torunoglu, E. Elsen, A. Karakas, Gauda Inc. (United States)

$76401 \mathrm{M}$ Source mask optimization for advanced lithography nodes [7640-56]

A. Poonawala, W. Stanton, C. Sawh, Synopsys, Inc. (United States)

\section{Part Two}

\section{SESSION 13 TOOLS}

7640 iN Towards ultimate optical lithography with NXT:1950i dual stage immersion platform [7640-57] T. Castenmiller, F. van de Mast, T. de Kort, C. van de Vin, M. de Wit, R. Stegen, S. van Cleef, ASML Netherlands B.V. (Netherlands) 
764010 Latest performance of immersion scanner S620D with the Streamlign platform for the double patterning generation [7640-58]

H. Kohno, Y. Shibazaki, J. Ishikawa, J. Kosugi, Y. Iriuchijima, M. Hamatani, Nikon Corp. (Japan)

7640 1P Performance of FlexRay: a fully programmable illumination system for generation of freeform sources on high NA immersion systems [7640-59]

M. Mulder, A. Engelen, O. Noordman, G. Streutker, B. van Drieenhuizen, C. van Nuenen, W. Endendijk, J. Verbeeck, W. Bouman, A. Bouma, R. Kazinczi, ASML (Netherlands); R. Socha, ASML Brion Technologies Inc. (United States); D. Jürgens, J. Zimmermann, B. Trauter, Carl Zeiss SMT AG (Germany); J. Bekaert, B. Laenens, IMEC vzw (Belgium); D. Corliss, G. Mclntyre, IBM Microelectronics (United States)

$76401 Q \quad$ High reliability ArF light source for double patterning immersion lithography [7640-60] R. Rokitski, T. Ishihara, R. Rao, R. Jiang, M. Haviland, T. Cacouris, D. Brown, Cymer, Inc. (United States)

7640 1R Advanced imaging with 1.35 NA immersion systems for volume production [7640-61] I. Bouchoms, J. Mulkens, S. de Putter, P. Gunter, R. de Graaf, M. Beems, E. Verdurmen, H. Jasper, ASML Netherlands B.V. (Netherlands); N. Dieckmann, Carl Zeiss SMT AG (Germany); F. Bornebroek, ASML Netherlands B.V. (Netherlands)

\section{POSTER SESSION: COMPUTATIONAL LITHOGRAPHY}

7640 is The impact of resist model on mask 3D simulation accuracy beyond 40-nm node memory patterns [7640-62]

K.-T. Chen, S.-S. Yeh, Y.-H. Hsieh, J.-C. N. Lai, Powerchip Semiconductor Corp. (Taiwan); S. A. Robertson, J. J. Biafore, S. Kapasi, A. Lin, KLA-Tencor Corp. (United States)

7640 1T Comparison of OPC models with and without 3D-mask effect [7640-63]

J.-H. Ser, T.-H. Park, M.-G. Jeong, E.-M. Lee, S.-W. Lee, C.-S. Suh, S.-W. Choi, C.-H. Park, J.-T. Moon, SAMSUNG Electronics Co., Ltd. (Korea, Republic of)

$76401 \mathrm{~V} \quad$ Virtual fab flow for wafer topography aware OPC [7640-64]

H.-J. Stock, Synopsys GmbH (Germany); L. Bomholt, Synopsys Switzerland LLC (Switzerland); D. Krüger, Synopsys GmbH (Germany); J. Shiely, H. Song, Synopsys Inc. (United States); N. Voznesenskiy, Riia (Estonia)

$76401 \mathrm{~V}$ Interlayer self-aligning process for 22-nm logic [7640-66] M. C. Smayling, Tela Innovations, Inc. (United States); S. Robertson, KLA-Tencor (United States); D. Lacey, Cavendish Kinetics, Inc. (United States); S. Kapasi, KLA-Tencor (United States)

$76401 \mathrm{~W} \quad$ Process window and integration results for full-chip model-based assist-feature placement at the 32-nm node and below [7640-67]

J. Li, Synopsys, Inc. (China); G. Luk-Pat, A. Poonawala, K. Lucas, B. Painter, Synopsys, Inc. (United States)

$76401 \mathrm{X} \quad$ The role of mask topography effects in the optimization of pixelated sources [7640-68] V. Domnenko, Synopsys, Inc. (Russian Federation); B. Küchler, T. Mülders, T. Schmöller, H.-J. Stock, G. Viehöver, Synopsys, Inc. (Germany) 
7640 IY Experimental study of effect of pellicle on optical proximity fingerprint for 1.35 NA immersion ArF lithography [7640-36]

L. Van Look, J. Bekaert, B. Laenens, G. Vandenberghe, IMEC (Belgium); J. Richter, K. Bubke, J. H. Peters, Advanced Mask Technology Ctr. GmbH (Germany); K. Schreel, M. Dusa, ASML B.V. (Netherlands)

$764012 \quad$ Achieving interferometric double patterning through wafer rotation [7640-70] P. Xie, N. V. Lafferty, B. W. Smith, Rochester Institute of Technology (United States)

764020 Novel ATHENA mark design to enhance alignment quality in double patterning with spacer process [7640-71]

L. W. Chen, M. Yang, E. Yang, T. H. Yang, K. C. Chen, C.-Y. Lu, Macronix International Co., Ltd. (Taiwan)

$764021 \quad$ Modeling of CD and placement error in multi-spacer patterning technology [7640-72] S. Babin, K. Bay, aBeam Technologies, Inc. (United States)

764022 LENS (lithography enhancement toward nano scale): a European project to support double exposure and double patterning technology development [7640-73]

P. Cantu, L. Baldi, P. Piacentini, Numonyx (Italy); J. Sytsma, ASML Netherlands (Netherlands); B. Le Gratiet, STMicroelectronics (France); S. Gaugiran, CEA-LETI (France); P. Wong, IMEC (Belgium); H. Miyashita, Dai Nippon Photomask Europe (Italy); L. R. Atzei, Lam Research Srl (Italy); X. Buch, JSR (Belgium); D. Verkleij, FEl (Netherlands); O. Toublan, Mentor Graphics (France); F. Perez-Murano, Ctr. Nacional de Microelectrónica (Spain); D. Mecerreyes, CIDETEC (Spain)

764023 Self-aligned double patterning process for 32/32-nm contact/space and beyond using 193 immersion lithography [7640-129]

B. Mebarki, L. Miao, Y. Chen, J. Yu, P. Blanco, J. Makeeff, J. Shu, C. Bencher, M. Naik, C. S. W. Ngai, Applied Materials, Inc. (United States)

\section{POSTER SESSION: FREEFORM AND SMO}

764024 Novel continuously shaped diffractive optical elements enable high efficiency beam shaping [7640-74]

Y. V. Miklyaev, W. Imgrunt, LIMO Lissotschenko Mikrooptik GmbH (Germany); V. S. Pavelyev, Image Processing Systems Institute (Russian Federation); D. G. Kachalov, Samara State Aerospace Univ. (Russian Federation); T. Bizjak, L. Aschke, V. N. Lissotschenko, LIMO Lissotschenko Mikrooptik GmbH (Germany)

764025 Advances in DOE modeling and optical performance for SMO applications [7640-75] J. Carriere, J. Stack, J. Childers, K. Welch, M. D. Himel, Tessera (United States)

764026 Abbe-PCA-SMO: microlithography source and mask optimization based on Abbe-PCA [7640-76]

J. H.-C. Chang, C.-P. C. Chen, National Taiwan Univ. (Taiwan); L. S. Melvin III, Synopsys (United States) 
764027 Optimization on illumination source with design of experiments [7640-77]

H. Hu, Y. Zou, Y. Deng, GLOBALFOUNDRIES Inc. (United States)

764028 Source-mask optimization (SMO): from theory to practice [7640-78]

T. Dam, V. Tolani, P. Hu, K.-H. Baik, L. Pang, B. Gleason, Luminescent Technologies, Inc.

(United States); S. D. Slonaker, J. K. Tyminski, Nikon Precision Inc. (United States)

\section{POSTER SESSION: LASER}

764029 Partial spatial coherence in an excimer-laser lithographic imaging system [7640-79]

A. Smith, A. Burvall, C. Dainty, National Univ. of Ireland, Galway (Ireland)

$76402 \mathrm{~A} \quad$ Flexible and reliable high power injection locked laser for double exposure and double patterning ArF immersion lithography [7640-80]

M. Yoshino, H. Umeda, H. Tsushima, H. Watanabe, S. Tanaka, S. Matsumoto, T. Onose,

H. Nogawa, Y. Kawasuji, T. Matsunaga, J. Fujimoto, H. Mizoguchi, Gigaphoton Inc. (Japan)

$76402 \mathrm{~B} \quad$ Laser bandwidth effect on overlay budget and imaging for the 45-nm and 32-nm technology nodes with immersion lithography [7640-81]

U. lessi, Numonyx (Italy); M. Kupers, Cymer B.V. (Netherlands); E. De Chiara, P. Rigolli,

Numonyx (Italy); I. Lalovic, Cymer, Inc. (United States); G. Capetti, Numonyx (Italy)

7640 2C Laser spectrum requirements for tight CD control at advanced logic technology nodes [7640-83]

R. C. Peng, H. J. Lee, J. Lin, Taiwan Semiconductor Manufacturing Corp. (Taiwan); A. Lin, KLA-Tencor Corp. (Taiwan); A. Chang, B. S.-M. Lin, Cymer Inc. (Taiwan)

7640 2D Lithography light source fault detection [7640-125]

M. Graham, E. Pantel, P. Nelissen, J. Moen, E. Tincu, W. Dunstan, D. Brown, Cymer, Inc. (United States)

\section{POSTER SESSION: LITHOGRAPHY OPTIMIZATION}

$76402 \mathrm{E} \quad$ Pattern deformation caused by deformed pellicle with ArF exposure [7640-85]

J.-H. You, Hanyang Univ. (Korea, Republic of); I. An, Nano-View Co. (Korea, Republic of);

H.-K. Oh, Hanyang Univ. (Korea, Republic of)

$764021 \quad$ Study for lithography techniques of hybrid mask shape of contact hole with 1.35-NA polarized illumination for 28-nm-node and below logic LSI [7640-88]

Y. Setta, K. Kobayashi, T. Chijimatsu, S. Asai, Fujitsu Microelectronics Ltd. (Japan)

\section{POSTER SESSION: MASK LAYOUT AND OPC}

7640 2J Applications of MoSi-based binary intensity mask for sub-40-nm DRAM [7640-89]

T.-S. Eom, E.-K. Shin, E.-H. Lee, Y.-J. Ryu, J.-T. Park, S. Koo, H.-J. Shin, S.-H. Hwang, H.-Y. Lim,

S. Park, K.-T. Sun, N.-J. Kwak, S. Park, Hynix Semiconductor Inc. (Korea, Republic of) 
7640 2K OMOG mask topography effect on lithography modeling of 32-nm contact hole patterning [7640-90]

L. Yuan, W. Zhou, GLOBALFOUNDRIES Singapore (Singapore); L. L. Zhuang, IBM

Microelectronics (United States); K. S. Yoon, SAMSUNG Electronics Co., Ltd. (Korea, Republic of); Q. Y. Lin, GLOBALFOUNDRIES Singapore (Singapore); S. Mansfield, IBM Microelectronics (United States)

$76402 \mathrm{~L} \quad$ Fast-converging iterative gradient decent methods for high pattern fidelity inverse mask design [7640-91]

J.-C. Yu, P. Yu, National Chiao Tung Univ. (Taiwan)

7640 2M Radial segmentation approach for contact hole patterning in 193-nm immersion lithography [7640-92]

M. L. Ling, National Univ. of Singapore (Singapore); G. S. Chua, S. K. Tan, GLOBALFOUNDRIES Singapore (Singapore); C. J. Tay, C. Quan, National Univ. of Singapore (Singapore); Q. Lin, GLOBALFOUNDRIES Singapore (Singapore)

7640 2N Binary mask optimization for forward lithography based on boundary layer model in coherent systems [7640-93]

X. Ma, G. R. Arce, Univ. of Delaware (United States)

$764020 \quad$ Improvement in process window aware OPC [7640-94]

X. Li, Synopsys Inc. (United States); Y. Kojima, H. Taoka, A. Moniwa, Renesas Technology Co. (Japan); M. St. John, Y. Ping, R. Brown, R. Lugg, S. Lee, Synopsys Inc. (United States)

7640 2P A non-delta-chrome OPC methodology for process models with three-dimensional mask effects [7640-95]

P. C. W. Ng, K.-Y. Tsai, National Taiwan Univ. (Taiwan); C.-H. Tang, National Taiwan Univ. (Taiwan) and Synopsys Taiwan Ltd. (Taiwan); L. Melvin, Synopsys, Inc. (United States)

$76402 \mathrm{~A}$ A new etch-aware after development inspection (ADI) technique for OPC modeling [7640-96]

J. Xue, J. Huang, A. Kazarian, B. Falch, Synopsys, Inc. (United States)

$76402 S \quad$ Wafer LMC accuracy improvement by adding mask model [7640-98]

W. C. Lo, Y. F. Cheng, M. J. Chen, P. Haung, United Microelectronics Corp. (Taiwan); S. Chang, Brion Technologies (United States); E. Tsujimoto, Dai Nippon Printing Co. (Japan)

$76402 \mathrm{ST} \quad$ Study of model based etch bias retarget for OPC [7640-99]

Q. Liu, R. Cheng, Semiconductor Manufacturing International Corp. (China); L. Zhang, Mentor Graphics Corp. (China)

$76402 \mathrm{U} \quad$ Intra field CD uniformity correction by Scanner Dose Mapper using Galileo mask transmission mapping as the CDU data source [7640-126]

G. S. Chua, GLOBALFOUNDRIES Singapore (Singapore); C. Eran, Carl Zeiss SMS (Israel);

S. K. Tan, B. I. Choi, T. H. Ng, P. L. Lua, GLOBALFOUNDRIES Singapore (Singapore);

O. Sharoni, G. Ben-Zvi, Carl Zeiss SMS (Israel) 
7640 2W Metamaterials for enhancement of DUV lithography (Best Student Paper Award) [7640-101] A. Estroff, N. V. Lafferty, P. Xie, B. W. Smith, Rochester Institute of Technology (United States)

$76402 Y$ Toward a consistent and accurate approach to modeling projection optics [7640-103]

D. Peng, P. Hu, V. Tolani, T. Dam, Luminescent Technologies (United States); J. Tyminski,

S. Slonaker, Nikon Precision Inc. (United States)

$76402 Z$ High fluence testing of optical materials for 193-nm lithography extensions applications [7640-127]

V. Liberman, S. Palmacci, G. P. Geurtsen, M. Rothschild, MIT Lincoln Lab. (United States);

P. A. Zimmerman, SEMATECH Inc. (United States)

\section{POSTER SESSION: MODELING}

764030 Stepwise fitting methodology for optical proximity correction modeling [7640-104]

A. Isoyan, J. Li, L. S. Melvin III, Synopsys, Inc. (United States)

764031 Automatic numerical determination of lateral influence functions for fast-CAD [7640-105] M. A. Miller, Univ. of California, Berkeley (United States); K. Yamazoe, Univ. of California, Berkeley (United States) and Canon Inc. (Japan); A. R. Neureuther, Univ. of California, Berkeley (United States)

764032 Aerial image model and application to aberration measurement [7640-106] A. Y. Bourov, L. Li, Z. Yang, F. Wang, L. Duan, Shanghai Micro Electronics Equipment Co., Ltd. (China)

764033 Methods for benchmarking photolithography simulators: part V [7640-107]

T. Graves, M. D. Smith, S. H. Kapasi, KLA-Tencor Corp. (United States)

764034 Selective inverse lithography methodology [7640-108]

C. Lim, V. Temchenko, Infineon Technologies Dresden GmbH and Co. OHG (Germany);

M. Niehoff, Mentor Graphics Corp. (Germany)

764035 CDU linear model based on aerial image principal components [7640-109]

Z. Yang, A. Y. Burov, L. Li, F. Wang, Z. Chu, Shanghai Micro Electronics Equipment Co., Ltd. (China)

764036 Impact of illumination on model-based SRAF placement for contact patterning [7640-110] J. L. Sturtevant, S. Jayaram, O. El-Sewefy, A. Dave, P. LaCour, Mentor Graphics Corp. (United States)

764037 A novel decomposition of source kernel for OPC modeling [7640-111] C. T. Hsuan, T. S. WU, F. Lo, E. Yang, T. H. Yang, K. C. Chen, C.-Y. LU, Macronix International Co., Ltd. (Taiwan)

764038 Methods for assessing empirical model parameters and calibration pattern measurements [7640-112]

X. Zhou, E. Khaliullin, L. Luan, Luminescent Technologies (United States) 
764039 A simplified reaction-diffusion system of chemically amplified resist process modeling for OPC [7640-113]

Y. Fan, Synopsys, Inc. (United States); M.-G. Jeong, J. Ser, S.-W. Lee, C. Suh, Samsung Electronics (Korea, Republic of); K.-I. Koo, S. Lee, Synopsys, Inc. (Korea, Republic of); I. Su, Synopsys, Inc. (Taiwan); L. Zavyalova, B. Falch, J. Huang, Synopsys, Inc. (United States); T. Schmoeller, Synopsys, Inc. (Germany)

7640 3A Improved process window modeling techniques [7640-114]

C. Zuniga, T. Tawfik, Mentor Graphics Corp. (United States)

POSTER SESSION: TOOLS AND PROCESS CONTROL

7640 3B Lithography cycle time improvements using short-interval scheduling [7640-115]

D. Norman, S. Watson, M. Anderson, S. Marteney, B. Mehr, Applied Materials, Inc. (United States)

7640 3C Topography-aware BARC optimization for double patterning [7640-117]

S. Liu, T. Fühner, F. Shao, Fraunhofer Institute for Integrated Systems and Device Technology (Germany); A. Barenbaum, Fraunhofer Institute for Integrated Systems and Device Technology (Germany) and Univ. of Erlangen-Nuremberg (Germany); J. Jahn, Univ. of Erlangen-Nuremberg (Germany); A. Erdmann, Fraunhofer Institute for Integrated Systems and Device Technology (Germany)

$76403 \mathrm{E}$ A novel method to reduce wafer topography effect for implant lithography process [7640-119]

L. Yuan, S. Bae, Y. F. Fu, A. Chen, H. P. Koh, Q. Y. Lin, GLOBALFOUNDRIES Singapore

(Singapore)

7640 3F Immersion BARC for hyper NA applications II [7640-121]

Y.-C. Huang, K.-L. Chuang, T.-J. Yeh, S. Wu, B. Lin, W.-L. Huang, B.-J. Lu, E. T. Liu, C. C. Yu, United Microelectronics Corp. (Taiwan); C. Lin, J. Y. YU, G. Prokopowicz, S. R. Kim, S. Wong, G. Barclay, The Dow Chemical Co. (United States)

$76403 \mathrm{G}$ Methods and challenges to extend existing dry 193-nm medium NA lithography beyond 90 nm [7640-122]

J. Schneider, A. Greiner, C. Lim, V. Temchenko, F. Braun, D. Kaiser, T. Hauck, I. Meusel,

D. Burmeister, S. Loehr, S. Volkland, A. Bauch, H. Kirbach, D. Sarlette, Infineon Technologies

Dresden GmbH (Germany); K. Thiede, Canon Deutschland GmbH (Germany)

$76403 \mathrm{H} \quad$ Examining reflectivity criterion for various ArF lithography [7640-123]

M.-F. Tsai, C.-C. Lin, W.-C. Chao, C.-T. WU, J.-C. Lai, Powerchip Semiconductor Corp.

(Taiwan)

7640 31 CD-uniformity for 45-nm NV memory on product-stack [7640-124]

U. Iessi, B. Colombo, Numonyx (Italy); J. Plauth, ASML Italy srl (Italy); B. Triulzi, E. De Chiara,

P. Canestrari, Numonyx (Italy) 
$76403 \mathrm{~J}$ Analysis of photoresist edge bead removal using laser light and gas [7640-128] V. Chaplick, E. Degenkolb, D. Elliott, K. Harte, R. Millman, Jr., M. Tardif, UVTech Systems, Inc. (United States)

Author Index 


\title{
Conference Committee
}

\author{
Symposium Chair
}

Christopher J. Progler, Photronics, Inc. (United States)

Symposium Cochair

Donis G. Flagello, Nikon Research Corporation of America (United States)

Conference Chair

Mircea V. Dusa, ASML MaskTools, Inc. (United States)

Conference Cochair

Will Conley, Freescale Semiconductor, Inc. (United States)

Program Committee

Pary Baluswamy, Micron Technology, Inc. (United States)

Andreas Erdmann, Fraunhofer-Institut für Integrierte System und Bavelementetechnologie (Germany)

Nigel R. Farrar, Cymer, Inc. (United States)

Roger H. French, DuPont Company (United States)

Tatsuhiko Higashiki, Toshiba Corporation (Japan)

Kurt R. Kimmel, Advanced Mask Technology Center GmbH Company KG (Germany)

Jongwook Kye, GLOBALFOUNDRIES Inc. (United States)

Tsai-Sheng Gau, Taiwan Semiconductor Manufacturing Company Ltd. (Taiwan)

Kafai Lai, IBM Corporation (United States)

SukJoo Lee, SAMSUNG Electronics Company, Ltd. (Korea, Republic of)

Wilhelm Maurer, Infineon Technologies AG (Germany)

Soichi Owa, Nikon Corporation (Japan)

Sam Sivakumar, Intel Corporation (United States)

Bruce W. Smith, Rochester Institute of Technology (United States)

Kazuhiro Takahashi, Canon Inc. (Japan)

Geert Vandenberghe, IMEC (Belgium) 
Session Chairs

1 Invited Session

Mircea V. Dusa, ASML MaskTools, Inc. (United States)

Will Conley, Freescale Semiconductor, Inc. (United States)

2 FreeForm and SMO

Soichi Inove, Toshiba Materials Company, Ltd. (Japan)

Sam Sivakumar, Intel Corporation (United States)

3 Double Patterning I

Tsai-Sheng Gau, Taiwan Semiconductor Manufacturing Company Ltd. (Taiwan)

Kazuhiro Takahashi, Canon Inc. (Japan)

4 Double Patterning II

Jung-Hoon Ser, SAMSUNG Electronics Company, Ltd. (Korea, Republic of)

Pary Baluswamy, Micron Technology, Inc. (United States)

5 Computational Lithography

Andreas Erdmann, Fraunhofer-Institut für Integrierte System und

Bavelementetechnologie (Germany)

Jongwook Kye, GLOBALFOUNDRIES Inc. (United States)

6 Polarization

Bruce W. Smith, Rochester Institute of Technology (United States)

Kurt G. Ronse, IMEC (Belgium)

$7 \quad$ Beyond $22 \mathrm{~nm}$

Sam Sivakumar, Intel Corporation (United States)

Soichi Inove, Toshiba Materials Company, Ltd. (Japan)

8 Tools and Process Resolution Extensions I

Kazuhiro Takahashi, Canon Inc. (Japan)

Tsai-Sheng Gau, Taiwan Semiconductor Manufacturing Company Ltd. (Taiwan)

9 Tools and Process Resolution Extensions II

Pary Baluswamy, Micron Technology, Inc. (United States)

Nigel R. Farrar, Cymer, Inc. (United States)

10 Mask, Layout, and OPC

Wilhelm Maurer, Infineon Technologies AG (Germany)

Jung-Hoon Ser, SAMSUNG Electronics Company, Ltd. (Korea, Republic of) 
$11 \quad$ Modeling

Sam Sivakumar, Intel Corporation (United States)

Nigel R. Farrar, Cymer, Inc. (United States)

12 Source-Mask Optimization

Jongwook Kye, GLOBALFOUNDRIES Inc. (United States)

Kafai Lai, IBM Corporation (United States)

13 Tools

Soichi Owa, Nikon Corporation (Japan)

Nigel R. Farrar, Cymer, Inc. (United States)

Poster Session: Computational Lithography

Bruce W. Smith, Rochester Institute of Technology (United States)

Poster Session: Double Patterning

Geert Vandenberghe, IMEC (Belgium)

Poster Session: FreeForm and SMO

Jongwook Kye, GLOBALFOUNDRIES Inc. (United States)

Tsai-Sheng Gau, Taiwan Semiconductor Manufacturing Company Ltd. (Taiwan)

Poster Session: Laser

Nigel R. Farrar, Cymer, Inc. (United States)

Poster Session: Lithography Optimization

Sam Sivakumar, Intel Corpration (United States)

Poster Session: Mask Layout and OPC

Wilhelm Maurer, Infineon Technologies AG (Germany)

Poster Session: Materials

Tsai-Sheng Gau, Taiwan Semiconductor Manufacturing Company Ltd. (Taiwan)

Poster Session: Modeling

Soichi Owa, Nikon Corporation (Japan)

Poster Session: Tools and Process Control

Kazuhiro Takahashi, Canon Inc. (Japan) 
Downloaded From: https://www.spiedigitallibrary.org/conference-proceedings-of-spie on 25 Apr 2023

Terms of Use: https://www.spiedigitallibrary.org/terms-of-use 